more time to tag tau for degradation by ubiquitination (8). However, the present study does not support this idea, since more CHIP/tau complexes formed in cells treated with Hsp90 siRNAs than in control-treated cells. Moreover, EC102-treated mice had much lower Hsp90 levels than did controls, in an apparent contradiction with the abrogation of the EC102 effect by Hsp90 siRNA in cultured cells.

The relative contributions of protein refolding, degradation, and aggregation to the neutralization of toxic tau are therefore far from being elucidated. Given that Hsp90 is involved in multiple signaling events unrelated to tau metabolism, it will be important to establish what potential side effects EC102 might have in long-term treatment. The key postulate of the study (EC102 specificity for Hsp90) may also need revisiting. EC102 has been reported to inhibit Hsp90 and not Hsp70 (16), but these studies do not address whether EC102 is specific for Hsp90. Downregulation of other components of the chaperone system also blocked the EC102-induced decrease in p-tau, raising the question whether this is due to the specific action of EC102 on Hsp90. These concerns notwithstanding, the notion that the chaperone machinery is a promising target for pharmacologic intervention in $\mathrm{AD}$ and other tauopathies has just received a robust boost.

Address correspondence to: Ronald Liem, Department of Pathology, Columbia University College of Physicians and Surgeons, 630 West 168th Street, New York, New York 10032, USA. Phone: (212) 305-4078; Fax: (212) 305-5498; E-mail: rk12@columbia.edu.

1. Young, J.C., Barral, J.M., and Ulrich Hartl, F. 2003. More than folding: localized functions of cytosolic chaperones. Trends Biochem. Sci. 28:541-547.

2. Ardley, H.C., and Robinson, P.A. 2004. The role of ubiquitin-protein ligases in neurodegenerative disease. Neurodegener. Dis. 1:71-87.

3. Muchowski, P.J., and Wacker, J.L. 2005. Modulation of neurodegeneration by molecular chaperones. Nat. Rev. Neurosci. 6:11-22.

4. Dou, F., et al. 2003. Chaperones increase association of tau protein with microtubules. Proc. Natl. Acad. Sci. U. S. A. 100:721-726.

5. Ardley, H.C., et al. 2003. Inhibition of proteasomal activity causes inclusion formation in neuronal and non-neuronal cells overexpressing Parkin. $\mathrm{Mol}$. Biol. Cell. 14:4541-4556

6. Ballinger, C.A., et al. 1999. Identification of CHIP, a novel tetratricopeptide repeat-containing protein that interacts with heat shock proteins and negatively regulates chaperone functions. Mol. Cell. Biol. 19:4535-4545

7. Connell, P., et al. 2001. The co-chaperone CHIP regulates protein triage decisions mediated by heat-shock proteins. Nat. Cell Biol. 3:93-96.
8. Young, J.C., Moarefi, I., and Hartl, F.U. 2001. Hsp90: a specialized but essential protein-folding tool. J. Cell Biol. 154:267-273.

9. Murata, S., Minami, Y., Minami, M., Chiba, T., and Tanaka, K. 2001. CHIP is a chaperone-dependent E3 ligase that ubiquitylates unfolded protein. EMBO Rep. 2:1133-1138.

10. Petrucelli, L., et al. 2004. CHIP and Hsp70 regulate tau ubiquitination, degradation and aggregation. Hum. Mol. Genet. 13:703-714.

11. Shimura, H., Schwartz, D., Gygi, S.P., and Kosik, K.S. 2004. CHIP-Hsc70 complex ubiquitinates phosphorylated tau and enhances cell survival. J. Biol. Chem. 279:4869-4876.

12. Sahara, N., et al. 2005. In vivo evidence of CHIP upregulation attenuating tau aggregation. J. Neurochem. 94:1254-1263.

13. Dickey, C.A., et al. 2006. Deletion of the ubiquitin ligase CHIP leads to the accumulation, but not the aggregation, of both endogenous phospho- and caspase-3-cleaved tau species. J. Neurosci. 26:6985-6996.

14. Dickey, C.A., et al. 2007. The high-affinity HSP90CHIP complex recognizes and selectively degrades phosphorylated tau client proteins. J. Clin. Invest. 117:648-658. doi:10.1172/JCI29715.

15. Biamonte, M.A., et al. 2006. Orally active purinebased inhibitors of the heat shock protein 90 . J. Med. Chem. 49:817-828.

16. Dickey, C.A., et al. 2006. HSP induction mediates selective clearance of tau phosphorylated at proline-directed Ser/Thr sites but not KXGS (MARK) sites. FASEB J. 20:753-755.

17. Panaretou, B., et al. 1998. ATP binding and hydrolysis are essential to the function of the Hsp90 molecular chaperone in vivo. EMBO J. 17:4829-4836.

18. Gong, C.X., Liu, F., Grundke-Iqbal, I., and Iqbal, K. 2005. Post-translational modifications of tau protein in Alzheimer's disease. J. Neural Transm. 112:813-838.

\title{
Salt, sodium channels, and SGK1
}

\author{
David Pearce ${ }^{1}$ and Thomas R. Kleyman²
}

\begin{abstract}
'Division of Nephrology, Department of Medicine, UCSF, San Francisco, California, USA. ${ }^{2}$ Renal-Electrolyte Division, Department of Medicine, and
\end{abstract} Department of Cell Biology and Physiology, University of Pittsburgh School of Medicine, Pittsburgh, Pennsylvania, USA.

\begin{abstract}
The hormone aldosterone increases extracellular fluid volume and blood pressure by activating epithelial $\mathrm{Na}^{+}$channels (ENaCs). Serum- and glucocorticoid-induced kinase 1 (SGK1) is an aldosterone-stimulated signaling molecule that enhances distal nephron $\mathrm{Na}^{+}$transport, in part by preventing the internalization of $\mathrm{ENaCs}$ from the plasma membrane. In this issue of the JCI, Zhang et al. demonstrate that SGK1 enhances transcription of the $\alpha$ subunit of $\mathrm{ENaC}$ by preventing histone methylation, providing an additional mechanism by which SGK1 increases $\mathrm{ENaC}$-mediated $\mathrm{Na}^{+}$transport in the distal nephron (see the related article beginning on page 773 ).
\end{abstract}

Nonstandard abbreviations used: AF9, ALL1-fused gene from chromosome 9; Dot1a, disruptor of telomeric silencing alternative splice variant a; $\mathrm{ENaC}$, epithelial $\mathrm{Na}^{+}$channel; GR, glucocorticoid receptor; $\mathrm{MR}$, mineralocorticoid receptor; MRE, mineralocorticoid response element; NR, nuclear receptor; SGK1, serum- and glucocorticoid-induced kinase 1.

Conflict of interest: The authors have declared that no conflict of interest exists.

Citation for this article: J. Clin. Invest. 117:592-595 (2007). doi:10.1172/JCI31538.
There are two general classes of $\mathrm{Na}^{+}$-selective ion channels in humans. The first, voltage-gated $\mathrm{Na}^{+}$channels, are transiently activated in response to modest degrees of membrane depolarization. Activation of these channels leads to a large but transient depolarization of the plasma membrane. In contrast, the second class, epithelial $\mathrm{Na}^{+}$ channels $(\mathrm{ENaCs})$, facilitate the bulk transit of $\mathrm{Na}^{+}$across an epithelial monolayer.
These channels are not strictly regulated by membrane voltage, and other mechanisms have been found to modulate their activity. The extent to which an $\mathrm{ENaC}$ is open and conducting $\mathrm{Na}^{+}$, a process referred to as open probability, is regulated by a number of factors, including phosphorylation, the presence of anionic phospholipids, and processing of $\mathrm{ENaC}$ extracellular domains by specific proteases (1-3). The number of channels present at the plasma membrane is also highly regulated, either by altering rates of channel biosynthesis and delivery to the plasma membrane or by channel retrieval from the plasma membrane (4-6).

$\mathrm{ENaCs}$ have key roles in the reabsorption of filtered $\mathrm{Na}^{+}$in the distal nephron of the kidney as well as in the regulation of $\mathrm{Na}^{+}$ content in the extracellular fluids, volume of extracellular fluids, and blood pressure. 


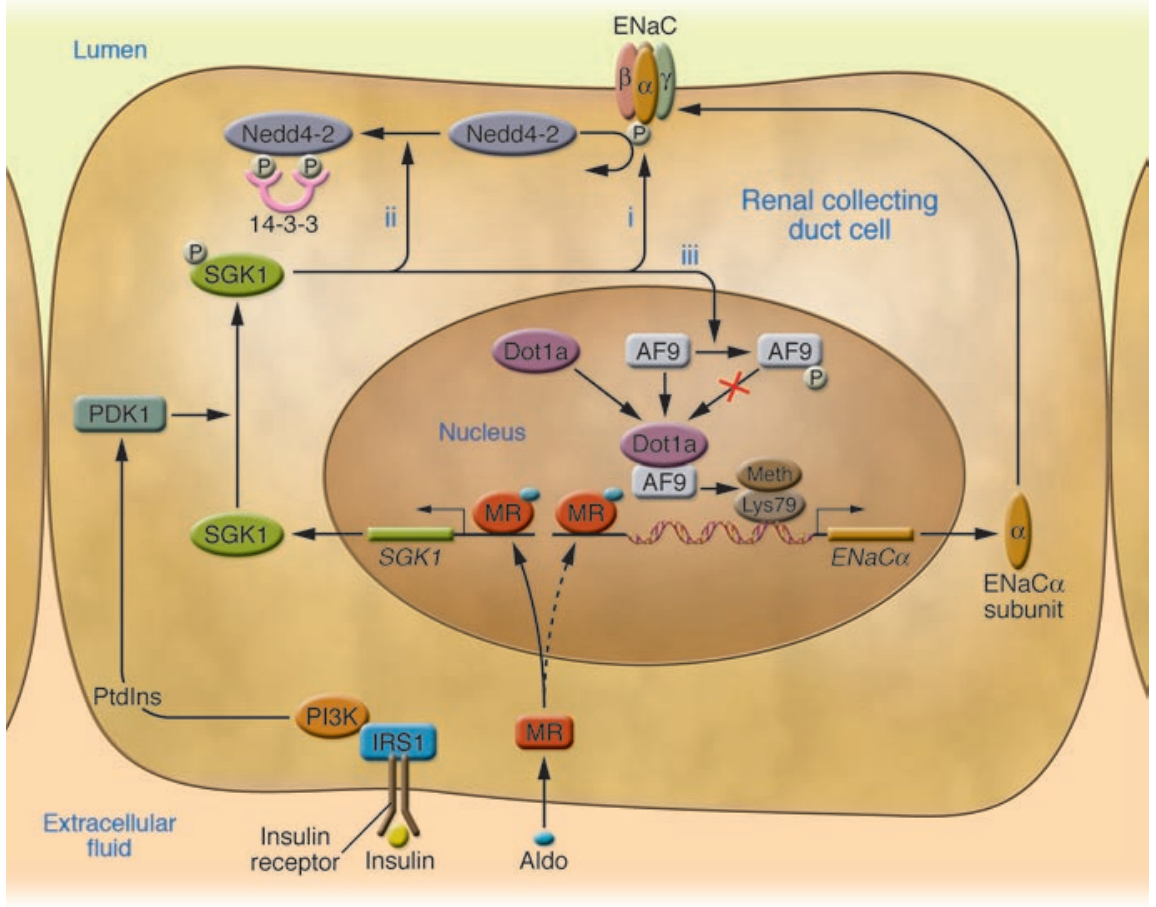

Figure 1

SGK1 acts at multiple levels to regulate ENaC-mediated $\mathrm{Na}^{+}$transport in renal collecting duct cells. At low aldosterone (Aldo) levels, $E N a C \alpha$ gene transcription is low because of tonic inhibition by a complex of Dot1a and AF9, which methylates Lys79 on histone H3. At the same time, Nedd4-2 is active and maintains plasma membrane ENaCs at low levels through ubiquitinylation, which results in channel internalization and degradation. With an elevation in aldosterone levels, activated MR translocates to the nucleus, binds to MREs in the SGK1 $5^{\prime}$ regulatory region, and rapidly stimulates SGK1 transcription (solid arrow). ENaC $\alpha$ is not initially responsive to aldosterone-activated MR because the Dot1a-AF9 complex maintains surrounding chromatin in a hypermethylated, stable state, which blocks entry of transcription factors, including MR itself, to their DNA-binding sites (dashed arrow). SGK1 catalytic activity is stimulated by PI3K-dependent generation of 3-phosphorylated phosphoinositides (Ptdlns), which activate phosphoinositide-dependent protein kinase 1 (PDK1) and ultimately SGK1 itself. SGK1 activates $\mathrm{ENaC}$ through phosphorylation of several key substrates: the channel itself (i), resulting in increased open probability; Nedd4-2 (ii), resulting in inhibition of Nedd4-2 through recruitment of inhibitory 14-3-3 chaperone proteins and subsequent decreased channel internalization and degradation; and AF9 (iii), resulting in inhibition of the Dot1a-AF9 complex and diminished H3 hypermethylation in the vicinity of the $\mathrm{ENaC} \alpha$ promoter. This latter effect, which is reported in this issue of the $\mathrm{JCl}(10)$, is predicted to enhance MR access to chromatin, binding to MREs, and stimulation of transcription. IRS1, insulin receptor substrate 1 ; Meth, methylation; $\alpha, \beta, \gamma, \mathrm{ENaC} \alpha$, $-\beta$, and $-\gamma$ subunits, respectively. Modified from ref. 10 and with permission from American Journal of Physiology. Renal Physiology (9).

The hormone aldosterone increases extracellular fluid volume, in large part via activation of ENaCs. Over the past decade, a variety of convergent mechanisms involved in the activation of ENaCs by aldosterone have been described.

\section{SGK1 is an aldosterone-regulated stimulator of ENaC}

Originally identified as a glucocorticoidregulated gene in a breast cancer cell line (7), serum- and glucocorticoid-induced kinase 1 (SGK1) is closely related to the Akt family of kinases and, like its cousins, nonhormonal signals. Regulators of this expression include glucocorticoids and mineralocorticoids, osmotic shock, folliclestimulating hormone, and TGF- $\beta$. Control of SGK1 activity is achieved primarily through a PI3K-dependent phosphorylation cascade; however, some regulators that act through this pathway, such as insulin, appear to have more of a pathophysiologic than a physiologic role.

\section{SGK1 regulates ENaC activity, trafficking, and gene transcription}

Some aspects of SGK1's mechanism of action are well defined, most notably its modulation of ENaC trafficking via inhibition of Nedd4-2, an ENaC inhibitor (reviewed in ref. 9). Well known for its role in Liddle's syndrome, Nedd4-2 is a Hect domain-containing ubiquitin ligase that interacts with the C-terminal tail of $\mathrm{ENaC}$ subunits and ubiquitinylates a set of $\mathrm{N}$-terminal lysines within channel subunits. Because Nedd4-2 triggers channel internalization and degradation, the effect of SGK1 in this context is one of disinhibition. Liddle's syndrome, which is one form of pseudohyperaldosteronism, results from an $\mathrm{ENaC}$ mutation that disrupts the interaction between channel and ligase and hence preempts SGK1-mediated inhibition of Nedd4-2. There is also some evidence to support the idea that SGK1 can regulate ENaC single-channel activities through direct phosphorylation of its $\alpha$ subunit, $\mathrm{ENaC \alpha}$ (3). In this issue of the JCI, Zhang et al. suggest what we believe to be a new way for SGK1 to regulate $\mathrm{ENaC} \alpha$ : through control of its gene transcription by altering local chromatin structure (10).

\section{Mineralocorticoid receptor-mediated gene regulation: early and late}

Like the gene encoding ENaC $\alpha$ itself, SGK1 is a classically regulated mineralocorticoid receptor (MR) target gene, with canonical mineralocorticoid response elements (MREs) driving its expression in response to MRs or glucocorticoid receptors (GRs) (11). However, SGK1 and ENaC $\alpha$ differ strikingly in the nature of their responses: SGK1 is an immediate early gene, the expression of which increases within minutes after cell exposure to corticosteroids and peaks within 1-2 hours. In contrast, $\mathrm{ENaC} \alpha$ expression increases slowly, with no change for the first several hours after exposure to aldosterone. Peak expression is not achieved for at least 12 hours. Moreover, prior evidence suggested that 
although direct $\mathrm{MR}$ regulation of $\mathrm{ENaC} \alpha$ gene transcription through its MREs was important, it was not the only mechanism involved, and that SGK1 might indeed be implicated (6). The basis for the differences in time course between immediate early genes such as SGK1 and late genes such as $E N a C \alpha$ has been mysterious. The current study by Zhang et al. (10) is exciting in that establishing a mechanistic connection between SGK1 and the activity of a histone methyl transferase complex provides one possible solution to this enigma.

\section{Histone methylation: an important locus of regulated gene expression}

Over the last several years, histone methylation has emerged as a central mechanism in the regulation of gene transcription (12). Through modification of arginines and lysines, primarily in the tails of histones $\mathrm{H} 3$ and $\mathrm{H} 4$, methylation can have complex stimulatory and inhibitory effects on gene transcription. Disruptor of telomeric silencing alternative splice variant a (Dot1a), the relevant methyl transferase in the control of ENaC expression, suppresses gene transcription by methylating Lys79 within the globular domain of histone $\mathrm{H} 3$ (i.e., histone H3 Lys79 methylation). This results (at least in yeast) in the recruitment of silencing proteins such as Sir2 and Sir3 and in chromatin stabilization (13-15).

Zhang, Kone, and colleagues demonstrated in earlier studies that aldosterone decreases histone $\mathrm{H} 3$ Lys79 methylation as it stimulates ENaC $\alpha$ mRNA levels and that the complex of Dot1a with ALL1-fused gene from chromosome 9 (AF9) mediates this effect $(16,17)$. This set the stage for their current work, which demonstrates the role of SGK1 in mediating this process and establishes a link between MR regulation of SGK1 on the one hand and of $\mathrm{ENaC} \alpha$ on the other (10). The authors demonstrate that both SGK1 and AF9 are associated with the ENaC $\alpha$ promoter and that Ser435 within AF9 is a target of SGK1. SGK1-dependent phosphorylation of AF9 reduced the interaction between Dot 1a and AF9, causing impaired association of Dot1a with the ENaC $\alpha$ promoter and concomitant histone $\mathrm{H} 3$ hypomethylation within specific subregions of the $\mathrm{ENaC} \alpha$ promoter. Furthermore, repression of the $\mathrm{ENaC} \alpha$ promoter by the Dot $1 \mathrm{a}-\mathrm{AF} 9$ complex was impaired with an AF9 Ser435Ala mutant. As expected based on these observations, mice maintained on a low-salt diet exhibited both low levels of renal $\mathrm{ENaC} \alpha$ protein expression and enhanced levels of AF9 phosphorylation. Mice lacking SGK1 expression exhibited reduced levels of AF9 phosphorylation and $\mathrm{ENaC} \alpha \mathrm{mRNA}$ levels when placed on a low-salt diet compared with wild-type mice fed the same diet.

The present data (10) provide strong evidence for the role of SGK1 in enhancing $\mathrm{ENaC} \alpha$ transcription by inducing AF9 phosphorylation and reducing the association of Dot1a with the ENaC $\alpha$ promoter, with an associated decrease in histone H3 Lys79 methylation. This effect of SGK1 is enhanced by aldosterone-dependent repression of both Dot1a and AF9 expression $(16,17)$. The SGK1-dependent increase in $\mathrm{ENaC} \alpha$ expression provides an additional mechanism by which SGK1 activates $\mathrm{ENaC}$ (Figure 1).

\section{Future directions}

One of the most interesting questions regarding the role of SGK1 in controlling $\mathrm{ENaC} \alpha$ expression is the teleological one: Why? Both ENaC $\alpha$ and SGK1 have in their regulatory regions canonical MREs that are functional and mediate MR activation of the gene. What would be the selection pressure that would favor the introduction of SGK1 itself as an intermediary in the control of $\mathrm{ENaC}$ expression? To us, the answer to this question lies in the economy, flexibility, and capacity for integration of inputs that this system provides. As noted above, SGK1 is an immediate early gene that responds rapidly to changes in aldosterone levels. In contrast, $E N a C \alpha$ is a late-response gene whose expression increases only after several hours of sustained hormone elevation. Because aldosterone rises and falls rapidly over a limited range during the course of a normal day in response to minor changes in dietary salt intake, SGK1 is similarly rising and falling and can rapidly influence $\mathrm{ENaC}$ trafficking and potentially single-channel activities. $\mathrm{ENaC} \alpha$ transcription under these conditions would not be expected to change; rather, the expression level would represent an integrated response to the combination of activated MRs (largely reflecting only the effect of aldosterone) and activated SGK1. Not only is the expression level of the latter regulated by inputs other than aldosterone (e.g., osmotic shock), its activity is controlled by the PI3K pathway and its vast array of regulatory inputs. Hence, the commitment of a cell to increase $\mathrm{ENaC}$ expression would presumably reflect a sustained commitment to enhance $\mathrm{Na}^{+}$reabsorption, such as one might see on a chronic low-salt diet. It will be of mechanistic and potentially pathophysiologic interest to examine the dependence of MR-stimulated $\mathrm{ENaC} \alpha$ expression on activated SGK1. The work of Zhang, Kone, and colleagues (10) predicts that activated SGK1 will enhance MR occupancy at the ENaC $\alpha$ promoter, synergize with MR in stimulating ENaCa expression, and alter the time course of aldosterone action. SGK1 inhibitors would be predicted to have the opposite effect and could be clinically useful as a potassium-sparing diuretic or for treatment of hypertension associated with the metabolic syndrome (also referred to as syndrome X). However, SGK1 has a variety of effects on glucose metabolism, growth, and proliferation in many cell types, including renal tubule and mammary epithelial cells and hepatocytes, and the potential clinical use of chemicals that modulate this important mediator must be approached with caution.

\section{Acknowledgments}

This work was supported by NIH grants DK056695 and DK054354.

Address correspondence to: Thomas R. Kleyman, Renal-Electrolyte Division, University of Pittsburgh, A919 Scaife Hall, 3550 Terrace Street, Pittsburgh, Pennsylvania 15261, USA. Phone: (412) 647-3121; Fax: (412) 648-9166; E-mail: kleyman@pitt.edu.

1. Hughey, R.P., et al. 2003. Maturation of the epithelial $\mathrm{Na}+$ channel involves proteolytic processing of the alpha- and gamma-subunits. J. Biol. Chem. 278:37073-37082.

2. Tong, Q., Gamper, N., Medina, J.L., Shapiro, M.S., and Stockand, J.D. 2004. Direct activation of the epithelial $\mathrm{Na}(+)$ channel by phosphatidylinositol 3,4,5-trisphosphate and phosphatidylinositol 3,4bisphosphate produced by phosphoinositide $3-\mathrm{OH}$ kinase. J. Biol. Chem. 279:22654-22663.

3. Diakov, A., and Korbmacher, C. 2004. A novel pathway of epithelial sodium channel activation involves a serum- and glucocorticoid-inducible kinase consensus motif in the C terminus of the channel's alpha-subunit. J. Biol. Chem. 279:38134-38142.

4. Butterworth, M.B., Edinger, R.S., Johnson, J.P., and Frizzell, R.A. 2005. Acute ENaC stimulation by cAMP in a kidney cell line is mediated by exocytic insertion from a recycling channel pool. J. Gen. Physiol. 125:81-101.

5. Chen, S.-Y., et al. 1999. Epithelial sodium channel regulated by aldosterone-induced protein sgk. Proc. Natl. Acad. Sci. U. S. A. 96:2514-2519.

6. Boyd, C., and Naray-Fejes-Toth, A. 2005. Gene regulation of $\mathrm{ENaC}$ subunits by serum- and glucocorticoid-inducible kinase-1. Am. J. Physiol. Renal Physiol. 288:F505-F512.

7. Webster, M.K., Goya, L., Ge, Y., Maiyar, A.C., and Firestone, G.L. 1993. Characterization of sgk, a novel member of the serine/threonine protein kinase gene family which is transcriptionally induced by glucocorticoids and serum. Mol. Cell. Biol. 13:2031-2040.

8. Wulff, P., et al. 2002. Impaired renal $\mathrm{Na}^{+}$reten- 
tion in the sgk1-knockout mouse. J. Clin. Invest. 110:1263-1268. doi:10.1172/JCI200215696.

9. Bhalla, V., Soundararajan, R., Pao, A.C., Li, H., and Pearce, D. 2006. Disinhibitory pathways for control of sodium transport: regulation of $\mathrm{ENaC}$ by SGK1 and GILZ. Am. J. Physiol. Renal Physiol. 291:F714-F721.

10. Zhang, W., et al. 2007. Aldosterone-induced Sgk1 relieves Dot1a-AF9-mediated transcriptional repression of epithelial $\mathrm{Na}^{+}$channel $\alpha$. J. Clin. Invest. 117:773-783. doi:10.1172/JCI29850.

11. Mick, V.E., et al. 2001. The alpha-subunit of the epithelial sodium channel is an aldosterone-induced transcript in mammalian collecting ducts, and this transcriptional response is mediated via distinct cis-elements in the 5 -flanking region of the gene. Mol. Endocrinol. 15:575-588.

12. Lee, D.Y., Teyssier, C., Strahl, B.D., and Stallcup, M.R. 2005. Role of protein methylation in regulation of transcription. Endocr. Rev. 26:147-170.

13. Feng, Q., et al. 2002. Methylation of H3-lysine 79 is mediated by a new family of HMTases without a SET domain. Curr. Biol. 12:1052-1058.

14. van Leeuwen, F., Gafken, P.R., and Gottschling, D.E. 2002. Dot $1 p$ modulates silencing in yeast by methylation of the nucleosome core. Cell. 109:745-756.
15. Ng, H.H., et al. 2002. Lysine methylation within the globular domain of histone $\mathrm{H} 3$ by Dot 1 is important for telomeric silencing and Sir protein association. Genes Dev. 16:1518-1527.

16. Zhang, W., et al. 2006. Aldosterone-sensitive repression of ENaCalpha transcription by a histone $\mathrm{H} 3$ lysine-79 methyltransferase. Am. J. Physiol., Cell Physiol. 290:C936-C946.

17. Zhang, W., Xia, X., Reisenauer, M.R., Hemenway, C.S., and Kone, B.C. 2006. Dot1a-AF9 complex mediates histone $\mathrm{H} 3$ Lys-79 hypermethylation and repression of ENaCalpha in an aldosterone-sensitive manner. J. Biol. Chem. 281:18059-18068.

\title{
HDL proteomics: pot of gold or Pandora's box?
}

\author{
Muredach P. Reilly ${ }^{1}$ and Alan R. Tall ${ }^{2}$ \\ ${ }^{1}$ Cardiovascular Institute, Department of Medicine, University of Pennsylvania Medical Center, Philadelphia, Pennsylvania, USA. \\ ${ }^{2}$ Division of Molecular Medicine, Department of Medicine, Columbia University Medical Center, New York, New York, USA.
}

\begin{abstract}
In this issue of the JCI, Vaisar et al. studied the proteome of HDL (see the related article beginning on page 746 ). They reveal, quite unexpectedly, that HDL is enriched in several proteins involved in the complement cascade, as well as in a variety of protease inhibitors, supporting the concept that HDL plays a role in innate immunity and in the regulation of proteolytic cascades involved in inflammatory and coagulation processes. The protein makeup of HDL also appears to be altered in patients with coronary artery disease. HDL proteomics is in its infancy, and preliminary findings will need to be confirmed using standardized approaches in larger clinical samples. However, this approach promises to better elucidate the relationship of HDL to atherosclerosis and its complications and could eventually help in the development of biomarkers to predict the outcome of interventions that alter HDL levels and functions.
\end{abstract}

The inverse relationship between plasma HDL-cholesterol (HDL-C) levels and atherosclerotic cardiovascular disease (CVD) provides the epidemiological basis for the widely accepted hypothesis that HDL is atheroprotective. Despite intense research, the underlying mechanisms of HDL atheroprotection remain incompletely understood. Indeed, recent clinical trials $(1,2)$ indicate the complexity of HDL physiology and the challenges in developing HDL therapies. HDL function, and benefit with a specific therapy, may depend more on

Nonstandard abbreviations used: $\mathrm{ABCA} 1, \mathrm{ABC}$ transporter $\mathrm{A} 1$; CAD, coronary artery disease; $\mathrm{CE}$, cholesterol ester; CETP, CE transfer protein; CVD, cardiovascular disease; HDL-C, HDL-cholesterol; ILLUMINATE, Investigation of Lipid Level Management to $\underline{\text { Understand Its }}$ Impact in ATherosclerotic Events; LCAT, lecithin:cholesterol acyltransferase; Lp-PLA 2 , lipoprotein-associated phospholipase $\mathrm{A}_{2}$; RCT, reverse cholesterol transport; SR-BI, scavenger receptor BI.

Conflict of interest: Muredach P. Reilly is in receipt of research funding from GlaxoSmithKline. Alan R. Tall has acted as a consultant to Bristol-Myers Squibb, Merck, Pfizer, Reddy Pharma, and Wyeth.

Citation for this article: J. Clin. Invest. 117:595-598 (2007). doi:10.1172/JCI31608. the molecular mechanism driving increases in HDL-C than on the absolute level of HDL-C (3). Some interventions that raise HDL-C levels may have no benefit and even promote atherosclerosis (4), while other therapies may reduce CVD without actually changing HDL-C levels (1). Overall, the epidemiological evidence suggests that the majority of mechanisms that result in higher HDL-C levels in vivo will provide atheroprotection; the question is how to identify such targets. This requires a shift in mindset toward assessing HDL in terms of its atheroprotective functions rather than just levels of cholesterol and its main apoprotein, APOAI.

\section{Proposed mechanisms of HDL atheroprotection}

Experimental studies, including limited work in humans, suggest several distinct but potentially overlapping HDL atheroprotective functions. These include reverse cholesterol transport (RCT) (5) and reductions in oxidative stress and in innate immune inflammation $(6,7)$.
RCT. The ability of HDL to promote RCT has been thought of as the major function of HDL for more than four decades $(8,9)$, although convincing demonstration of this process in vivo has only emerged in the past few years (5). In atherosclerosis the primary cell that is loaded with cholesterol is the arterial macrophage - therefore, it may make more sense to conceptualize RCT in terms of macrophage cholesterol efflux potential or "macrophage RCT" rather than in terms of total peripheral tissue cholesterol RCT $(5,9)$.

The first step in macrophage RCT is efflux of cholesterol from arterial macrophages, a highly regulated process involving specific transporters including $A B C$ transporter $\mathrm{A} 1$ (ABCA1) and ABCG1 (5). ABCA1 facilitates efflux of cholesterol and phospholipids (including oxidized phospholipids) to lipid-poor APOAI, whereas ABCG1-mediated cholesterol efflux to more mature HDL particles (10) is enhanced by lecithin:cholesterol acyltransferase (LCAT) and APOE in HDL (11) and may be in part be responsible for the "passive cholesterol efflux" characterized by Rothblat, Phillips, and coworkers (12). Expression of both transporters is upregulated in macrophages by oxysterols that activate the nuclear hormone receptor liver X receptors (LXRs) and directly target the promoters of theses genes. Macrophage expression of both ABCA1 and ABCG1 enhances macrophage cholesterol efflux and protects against experimental atherosclerosis.

Following efflux to HDL and esterification by LCAT, transport of cholesterol to the liver is mediated directly by HDL hepatic receptors, including scavenger 